\title{
Role of Intravenous immunoglobulin in Non- Immunocompromised children With Severe Adenovirus Pneumonia: A Retrospective Observational Study
}

\section{Huifeng Fan}

Guangzhou Women and Children's Medical Center https://orcid.org/0000-0002-1127-4256

\section{Chen Chen}

Guangzhou Women and Children's Medical Center

\section{Xuehua Xu}

Guangzhou Women and Children's Medical Center

\section{Senqiang Zeng}

Guangzhou Women and Children's Medical Center

Genquan Yin

Guangzhou Women and Children's Medical Center

Wenhui Jiang

Guangzhou Women and Children's Medical Center

Gen Lu ( $\square$ lugen5663330@sina.com)

Guangzhou Medical University https://orcid.org/0000-0001-9702-6167

\section{Research article}

Keywords: severe adenovirus pneumonia, intravenous immunoglobulin, children

Posted Date: October 9th, 2020

DOI: https://doi.org/10.21203/rs.3.rs-88010/v1

License: @ (i) This work is licensed under a Creative Commons Attribution 4.0 International License. Read Full License 


\section{Abstract}

Background: Adenovirus pneumonia is a pulmonary infectious disease commonly occurring in children, and severe cases can lead to death and sequelae. Here, we aimed to observe the therapeutic timing and dosage of intravenous immunoglobulin(IVIg) in non-immunocompromised children with severe adenovirus pneumonia.

Methods: This retrospective observational study investigated severe adenovirus pneumonia treated with IVIg in nonimmunocompromised pediatric patients at a tertiary hospital in 2019. Participants were classified as early presenters (5-10 days of illness course) and later presenters (11-15 days) according to the timing of IVIg treatment. Patients' clinical data were then analyzed in terms of different dosages of IVIg administration.

Results: Among 202 patients enrolled, 128 were early presenters and 74 were later presenters during the study period. The later presenters had longer fever duration, more incidences of fungal coinfections, more demands for mechanical ventilation, and higher incidence of bronchiectasis than early presenters $(P<0.05)$. For early presenters, no statistically significant differences in demands for advanced life support, outcomes and sequelae were observed between the two different dosage groups ( $P>0.05$ ). For later presenters, shorter fever duration and lower usage of extracorporeal membrane oxygenation (ECMO) were observed in the high-dosage group than that in the low-dosage group $(P<0.05)$. The incidence of post-infectious bronchiolitis obliterans (PIBO) and bronchiectasis was not significantly different between the two groups ( $P>0.05$ ). The incidence of adverse events was $6.62 \%$ during IVIg infusion, showing no significant difference between the two groups ( $P>0.05)$.

Conclusions: Early medical care and treatment with IVIg are very important to improve the prognosis of nonimmunocompromised children with severe adenovirus pneumonia. For later presenters, children with severe conditions may benefit from a high IVIg dosage.

\section{Background}

Human adenoviruses (HAdVs) are non-enveloped, double-stranded DNA viruses that can cause respiratory tract diseases in children[1]. They are estimated to cause $5-10 \%$ of pediatric respiratory tract infections (RTIs) [1, 2]. Adenovirus respiratory tract infections have been known to be usually mild and self-limiting in a nonimmunocompromised host; however, previous studies have described fatal cases of severe adenovirus pneumonia caused by particular serotypes, even in non-immunocompromised patients $[3,4]$. About 70 adenovirus serotypes are divided into seven subgroups or species (A through $\mathrm{G}$ ), and pneumonia is usually caused by HAdV-3, 7, and 21 , with HAdV-7 being associated with serious respiratory illness in infants and young children[5, 6]. A 14-60\% risk of developing long-term respiratory aftereffects such as post-infectious bronchiolitis obliterans (PIBO) and bronchiectasis was observed in severe adenovirus pneumonia [7].

Currently, no specific therapy has demonstrated efficacy in the treatment of adenovirus pneumonia[1]. Several studies have shown that cidofovir (CDV) has the greatest in vitro activity against adenovirus among currently available antiviral agents and is the preferred therapeutic agent[8]. However, adverse effects of CDV include nephrotoxicity, myelosuppression, and uveitis, which limit its application in healthy children[1]. Currently, CDV is only used intravenously in many pediatric transplant clinics for the treatment of adenovirus infections[9]. Data on the response to CDV are limited in non-immunocompromised children. Therefore, intravenous immunoglobulin (IVlg) as an adjuvant therapy for severe adenovirus pneumonia has received increasing attention from clinicians. Several reports have recently re-emphasized the potential use of antibodies in the form of IVIg as an anti-infectious agent in severe adenovirus infections $[10,11]$. Exposure of such donors to their unique environment increased the IVIg dosage, 
consisting a diverse repertoire of immunoglobulin molecules that possess a wide spectrum of antibacterial and antiviral specificities[12-14]. However, consensus on indications, timing, and dosage of IVIg among clinicians is limited in children with severe adenovirus pneumonia.

Accordingly, we primarily evaluated the timing of IVIg for the treatment of severe adenovirus pneumonia. Our data were analyzed in terms of the efficacy and safety of different IVIg dosages to prevent disease progression and mortality.

\section{Methods}

\section{Study population and ethics}

One thousand four hundred and five pediatric patients with adenovirus pneumonia on admission who attended Guangzhou Women and Children's Medical Center from January 1, 2019, to January 1, 2020 were eligible for this study. The World Health Organization has published evidence-based guidelines in diagnosing pneumonia in children[15]. The evidence of adenovirus infection was identified by positive multiplex polymerase chain reaction (PCR) for HAdVs from nasopharyngeal swabs, sputum, and bronchial alveolar lavage fluid. This study protocol was conducted in accordance with the Declaration of Helsinki and approved by the Ethics Committee of Guangzhou Women and Children's Medical Center of Guangzhou Medical University. All patients provided written informed consent for the use of their clinical and laboratory data from their medical reports.

\section{Data Collection and Definitions}

Inclusion criteria for the IVIg trial in the treatment of severe adenovirus pneumonia were as follows:

1) 28 days $<$ age $\leq 18$ years

2) Definition of severe cases[15]: cough or difficulty in breathing, combined with at least one of the following: a) central cyanosis or oxygen saturation of < $90 \%$ on pulse oximetry; b) severe respiratory distress (e.g., grunting, very severe chest indrawing); c) signs of pneumonia with a general danger sign (inability to breastfeed or drink, lethargy or reduced level of consciousness, convulsions); and d) auscultatory findings of decreased or bronchial breath sounds or signs of pleural effusion or empyema.

3) Treatment with IVIg administration

4) Informed consent was signed by the guardians of study participants.

Exclusion criteria for the IVIg trial in the treatment of adenovirus pneumonia were as follows:

1) Admission to pediatric intensive care unit (PICU) before IVIg administration;

2) Known or suspected active tuberculosis, severe concomitant disease (chronic pulmonary disease such as asthma, severe cardiovascular disease, neoplasia, and kidney or liver disease), and immunocompromised state (primary immunodeficiency, acquired immunodeficiency syndrome, and immunosuppressive medications before admission).

Clinical information of children with severe adenovirus pneumonia was collected during the study period.

\section{Study Design}


This retrospective, observational study was conducted in accordance with the Strengthening the Reporting of Observational Studies in Epidemiology (STROBE) guidelines. To reduce time-dependent bias, stratification analysis was applied to patients with severe adenovirus pneumonia according to the charged time with the timing of IVIg administration. The time, measured in days since the onset of fever (temperature, $\geq 37.5^{\circ} \mathrm{C}$ ) was the initial symptom of adenovirus pneumonia. According to fever onset, patients were classified into two groups: 1) early presenters: IVIg administration in 5-10 days of illness course; and 2) later presenters: IVlg administration in 11-15 days of illness course. Clinical manifestations and outcomes were then compared between the two layers.

Next, patients from both layers were divided into two groups according to different IVIg dosages: 1) high-dosage group ( $1 \mathrm{~g} / \mathrm{kg} /$ day for 2 days); and 2) low-dosage group (0.4-0.5 g/kg/day for 3-5 days). Demographic characteristics, laboratory findings, image characteristics, and treatment before IVIg administration were compared between the two groups in each layer.

The observation end-points consisting of two parts were collected and compared between the two groups. Primary end-points were as follows: 1) advanced life support: mechanical ventilation, continuous blood purification (CBP), extracorporeal membrane oxygenation (ECMO); 2) fever duration; 3) length of stay (LOS); and 4) mortality. Secondary end-points (long-term sequelae) were 1) post-infectious bronchiolitis obliterans (PIBO) and 2) bronchiectasis.

Finally, we compared the incidence of adverse events of IVIg infusion according to different dosages, including flushing, headache, fever, chills, nausea and vomiting, hypotension, nephrotoxicity, myalgias, anaphylactic shock, thromboembolic events, hemolysis, fatigue and lethargy[16-18].

\section{Statistical Analysis}

Statistical analyses were performed using GraphPad Prism 8.0 (GraphPad Software Inc., San Diego, CA, USA). As data of continuous variables were skewed distribution, medians with ranges were used to express the summarized data. The nonparametric Mann-Whitney test was used for two-group analysis of continuous variables. Categorical variables were assessed using Fisher's exact test. A $P$-value of $<0.05$ was considered statistically significant.

\section{Results}

\section{Patients' Baseline Characteristics}

A total of 202 patients (123 male and 79 female) were enrolled in the present study (Fig. 1), with the median age of 18 (range, 1-144) months (Table 1). Among them, 128 patients (63.37\%) were administered IVIg in 5-10 days of illness course, and 74 (36.63\%) in 11-15 days of illness course. The primary signs and symptoms of severe adenovirus pneumonia were fever, cough, and tachypnea in all patients. The median fever duration was 13 days at baseline and ranged from 3 to 58 days. The primary HAdV types of patients were HAdV-7 (128/202, 63.37\%) and HAdV-3(27/202, 13.37\%). Among the coinfections, the most common pathogens were bacteria $(65 / 202,32.18 \%)$ and Mycoplasma pneumoniae (M. pneumoniae) (52/202, 25.74\%). The first major pulmonary complications were consolidation (150/202, 74.26\%) (Fig. 2a-C), and the second was pleural effusion and empyema (62/202, 30.69\%). Acute respiratory distress syndrome (ARDS)[19] was the most common systemic complication (35/202, 17.33\%). Other less common complications included sepsis shock[20] (27/202, 13.37\%), multiple organ dysfunction syndrome (MODS) [21](19/202, 9.41\%), and hemophagocytic lymphohistiocytosis (HLH) [22](3/202, 1.49\%). PIBO was the most common sequela after a severe adenovirus pneumonia (Fig. 2d-f), affecting up to one-fifth of survivors (48/202, $23.76 \%)$. The rate of bronchiectasis was low (9/202, 4.46\%). The median LOS was 15 (range, 1-70 days), and the overall mortality due to severe adenovirus pneumonia was approximately $4.95 \%(10 / 202)$. 
Table 1

Stratification analysis of children with severe adenovirus pneumonia according to the timing of IVIg administration in the study

\begin{tabular}{|c|c|c|c|c|}
\hline Variables & $\begin{array}{l}\text { Total } \\
(n=202)\end{array}$ & $\begin{array}{l}\text { Early presenters } \\
(n=128)\end{array}$ & $\begin{array}{l}\text { Later presenters } \\
(\mathrm{n}=74)\end{array}$ & $\begin{array}{l}P \\
\text { value }\end{array}$ \\
\hline \multicolumn{5}{|l|}{ Demographic } \\
\hline Age (months), median(range) & $18(1-144)$ & 18(1-132) & $18(4-144)$ & 0.4070 \\
\hline Gender, male (\%) & 123(60.89) & $74(57.81)$ & $49(66.22)$ & 0.2950 \\
\hline \multicolumn{5}{|l|}{ Signs and Symptoms } \\
\hline Fever, No. (\%) & $202(100)$ & $128(100.00)$ & $74(100.00)$ & 1.0000 \\
\hline Cough, No. (\%) & 202(100) & $128(100.00)$ & $74(100.00)$ & 1.0000 \\
\hline Tachypnea, No. (\%) & $202(100)$ & $128(100.00)$ & $74(100.00)$ & 1.0000 \\
\hline Wheezing, No. (\%) & $48(23.76)$ & $30(23.44)$ & $18(24.32)$ & 0.9999 \\
\hline Moist crackles, No. (\%) & 178(88.12) & $111(86.72)$ & $67(90.54)$ & 0.5026 \\
\hline Hepatosplenomegaly, No. (\%) & $30(14.51)$ & $18(14.06)$ & $12(16.22)$ & 0.6857 \\
\hline $\begin{array}{l}\text { Change in level of consciousness }{ }^{a} \text {, } \\
\text { No. (\%) }\end{array}$ & $49(24.26)$ & $27(21.09)$ & $22(29.73)$ & 0.1709 \\
\hline Digestive symptoms, No. (\%) & $53(26.24)$ & $37(28.91)$ & $16(21.62)$ & 0.3197 \\
\hline \multicolumn{5}{|l|}{ Laboratory findings ${ }^{a}$} \\
\hline $\begin{array}{l}\text { White blood cells }\left(\times 10^{\wedge} 9 / L\right)(5-12) \text {, } \\
\text { median(range) }\end{array}$ & $7.15(1.30-30.60)$ & $7.30(1.30-25.30)$ & $6.90(1.50-30.60)$ & 0.4060 \\
\hline $\begin{array}{l}\text { Neutrophil }\left(\times 10^{\wedge} 9 / \mathrm{L}\right)(5-12) \text {, } \\
\text { median(range) }\end{array}$ & $3.82(0.48-22.53)$ & $\begin{array}{l}4.035(0.48- \\
21.84)\end{array}$ & $3.42(0.64-22.53)$ & 0.4357 \\
\hline $\begin{array}{l}\text { Hemoglobin(g/L)(105- } \\
\text { 145), median(range) }\end{array}$ & $105.50(56-145)$ & $108(56-145)$ & $100.5(67-141)$ & 0.0223 \\
\hline $\begin{array}{l}\text { Platelet }\left(\times 10^{\wedge} 12 / L\right)(5-12), \\
\text { median(range) }\end{array}$ & $255.50(15-1180)$ & $252(70-1180)$ & $281(15-766)$ & 0.8630 \\
\hline $\mathrm{HsCRP}(\mathrm{mg} / \mathrm{L})(<5)$, median(range) & $\begin{array}{l}18.70(0.46- \\
222.22)\end{array}$ & $\begin{array}{l}\text { 19.02(0.46- } \\
194.23)\end{array}$ & $17.80(0.5-222.22)$ & 0.5700 \\
\hline LDH(U/L) (159-322), median(range) & $688(210-4836)$ & $625(210-4836)$ & $845.5(260-4570)$ & 0.0101 \\
\hline Albumin(g/L)(40-50), median(range) & $\begin{array}{l}32.20(20.20- \\
47.40)\end{array}$ & $\begin{array}{l}33.75(21.22- \\
47.40)\end{array}$ & $\begin{array}{l}30.50(20.20- \\
45.20)\end{array}$ & 0.0066 \\
\hline $\mathrm{PaO}_{2}(\mathrm{kPa})(\geq 10.9)$, median (range) & $\begin{array}{l}8.825(3.30- \\
28.00)\end{array}$ & $9.10(4.51-28.00)$ & 8.53(3.30-20.07) & 0.3672 \\
\hline $\begin{array}{l}\mathrm{PaCO}_{2}(\mathrm{kPa})(4.66-5.99), \text { median } \\
\text { (range) }\end{array}$ & $4.87(3.20-13.20)$ & $4.84(3.20-6.74)$ & $4.96(3.61-13.20)$ & 0.1264 \\
\hline HAdV-3, No. (\%) & $27(13.37)$ & $15(11.72)$ & $12(16.22)$ & 0.3951 \\
\hline HAdV-7, No. (\%) & 128(63.37) & $77(60.16)$ & $51(68.92)$ & 0.2288 \\
\hline
\end{tabular}




\begin{tabular}{|c|c|c|c|c|}
\hline Variables & $\begin{array}{l}\text { Total } \\
(n=202)\end{array}$ & $\begin{array}{l}\text { Early presenters } \\
(n=128)\end{array}$ & $\begin{array}{l}\text { Later presenters } \\
(n=74)\end{array}$ & $\begin{array}{l}P \\
\text { value }\end{array}$ \\
\hline \multicolumn{5}{|l|}{ Immunologic assays ${ }^{a}$} \\
\hline $\begin{array}{l}\text { Serum } \lg E(I U / M L)(0-200), \\
\text { median(range) }\end{array}$ & $85(5-3890)$ & $80.50(5-3890)$ & $98(6-1110)$ & 0.3278 \\
\hline $\begin{array}{l}\text { Serum IgM(g/L)(0.42-1.46), } \\
\text { median(range) }\end{array}$ & $1.31(0.33-6.06)$ & $1.24(0.33-6.06)$ & $1.45(0.3-4.62)$ & 0.2135 \\
\hline $\begin{array}{l}\text { Serum IgA(g/L)(0.49-2.29), } \\
\text { median(range) }\end{array}$ & $0.68(0.07-6.08)$ & $0.63(0.09-6.08)$ & $0.74(0.07-3.68)$ & 0.3622 \\
\hline $\begin{array}{l}\text { Serum lgG(g/L)(6.36-13.24), } \\
\text { median(range) }\end{array}$ & $9.70(1.23-32.70)$ & $9.47(2.50-32.70)$ & $10.60(1.23-31.00)$ & 0.2883 \\
\hline $\begin{array}{l}\mathrm{CD} 3+4+\text { Abs(cells/ul)(345-2350), } \\
\text { median(range) }\end{array}$ & $\begin{array}{l}402.925(345- \\
2350)\end{array}$ & $\begin{array}{l}451.70(124.86- \\
1733.05)\end{array}$ & $\begin{array}{l}435.17(113.80- \\
2274.12)\end{array}$ & 0.6922 \\
\hline $\begin{array}{l}\mathrm{CD} 3+8+\mathrm{Abs}(\mathrm{cells} / \mathrm{ul})(314-2080) \\
\text { median(range) }\end{array}$ & $\begin{array}{l}300.25(106.31- \\
2001.93)\end{array}$ & $\begin{array}{l}279.09(106.31- \\
2001.93)\end{array}$ & $\begin{array}{l}306.69(133.45- \\
1146.94)\end{array}$ & 0.7331 \\
\hline $\begin{array}{l}\text { CD19+ Abs(cells/ul)(240-1317), } \\
\text { median(range) }\end{array}$ & $\begin{array}{l}357.045(47.90- \\
2267.10)\end{array}$ & $\begin{array}{l}361.47(47.90- \\
871.86)\end{array}$ & $\begin{array}{l}336.24(50.09- \\
2267.10)\end{array}$ & 0.2318 \\
\hline Th/Ts(\%)(0.47-2.05), median(range) & $1.275(0.35-6.29)$ & $1.27(0.59-6.29)$ & $1.71(0.35-3.62)$ & 0.6057 \\
\hline $\begin{array}{l}\text { NKAbs(cells/ul)(345-2350), } \\
\text { median(range) }\end{array}$ & $\begin{array}{l}50.09(2.92- \\
775.44)\end{array}$ & $\begin{array}{l}48.00(2.92- \\
775.44)\end{array}$ & $\begin{array}{l}50.09(8.08- \\
727.49)\end{array}$ & 0.7208 \\
\hline NBT test, No. (\%) & $0(0.00)$ & $0(0.00)$ & $0(0.00)$ & 1.0000 \\
\hline \multicolumn{5}{|l|}{ Co-infection ${ }^{b}$} \\
\hline Backteria, No. (\%) & $65(32.18)$ & $42(32.81)$ & 23(31.08) & 0.8762 \\
\hline Virus(except for HAdVs), No. (\%) & $40(19.80)$ & $23(17.97)$ & 17(22.97) & 0.4640 \\
\hline Mycoplasma pneumoniae, No. (\%) & $52(25.74)$ & $30(23.44)$ & $22(29.33)$ & 0.4057 \\
\hline Fungi, No. (\%) & $29(14.36)$ & $13(10.16)$ & $16(21.62)$ & 0.0361 \\
\hline \multicolumn{5}{|l|}{ Pulmonary complications ${ }^{b}$} \\
\hline Consolidation, No. (\%) & $150(74.26)$ & $93(72.66)$ & $57(77.03)$ & 0.6166 \\
\hline Pleural effusion, No. (\%) & $62(30.69)$ & $37(28.91)$ & 25(33.78) & 0.5273 \\
\hline $\begin{array}{l}\text { Pneumothorax and } \\
\text { pneumomediastinum, No. (\%) }\end{array}$ & $13(6.44)$ & $6(4.69)$ & $7(9.46)$ & 0.2353 \\
\hline \multicolumn{5}{|l|}{ Systemic complications } \\
\hline ARDS, №. (\%) & $35(17.33)$ & $20(15.63)$ & $15(20.27)$ & 0.4426 \\
\hline Sepsis shock, No. (\%) & $27(13.37)$ & 15(11.72) & $12(16.22)$ & 0.3951 \\
\hline MODS, No. (\%) & 19(9.41) & $12(9.38)$ & $7(9.46)$ & 0.9999 \\
\hline HLH, No. (\%) & $3(1.49)$ & $2(1.56)$ & $1(1.35)$ & 0.9999 \\
\hline
\end{tabular}




\begin{tabular}{|c|c|c|c|c|}
\hline Variables & $\begin{array}{l}\text { Total } \\
(n=202)\end{array}$ & $\begin{array}{l}\text { Early presenters } \\
(n=128)\end{array}$ & $\begin{array}{l}\text { Later presenters } \\
(n=74)\end{array}$ & $\begin{array}{l}P \\
\text { value }\end{array}$ \\
\hline \multicolumn{5}{|l|}{ Treatment(Before IVIg) } \\
\hline Oxygen, No. (\%) & 202(100) & $128(100.00)$ & 74(100.00) & 1.0000 \\
\hline Antibotics, No. (\%) & 125(61.88) & $78(60.94)$ & $47(63.51)$ & 0.7649 \\
\hline Systemic corticosteroid, No. (\%) & 132(65.35) & $86(67.19)$ & $46(62.16)$ & 0.5398 \\
\hline \multicolumn{5}{|l|}{$\begin{array}{l}\text { Pediatric Advanced Life Support(After } \\
\text { IVlg) }\end{array}$} \\
\hline Mechanical ventilation, №. (\%) & $51(21.25)$ & $26(20.31)$ & 25(33.78) & 0.0435 \\
\hline CBP, No. (\%) & 11(5.45) & $5(3.91)$ & $6(8.11)$ & 0.2157 \\
\hline ECMO, No. (\%) & $15(7.43)$ & $9(7.03)$ & $6(8.11)$ & 0.7860 \\
\hline \multicolumn{5}{|l|}{ Sequelae } \\
\hline PIBO, No. (\%) & $48(23.76)$ & $28(21.88)$ & $20(27.03)$ & 0.4928 \\
\hline Bronchiectasis, No. (\%) & $9(4.46)$ & $2(1.56)$ & $7(9.46)$ & 0.0129 \\
\hline \multicolumn{5}{|l|}{ Outcomes } \\
\hline Fever duration(days), median(range) & $13(3-58)$ & $11(3-58)$ & $18(6-44)$ & $<_{0.0001}$ \\
\hline Length of stay(days), median(range) & $15(1-70)$ & $13(4-70)$ & $17(1-60)$ & 0.1235 \\
\hline Mortality, No. (\%) & $10(4.95)$ & $4(3.31)$ & $6(8.11)$ & 0.1756 \\
\hline \multicolumn{5}{|c|}{$\begin{array}{l}\text { Abbreviation: HsCRP: high-sensitivity C-reactive protein; LDH: lactate dehydrogenase; NBT: nitroblue tetrazolium; } \\
\text { ARDS:acute respiratory distress syndrome; SIRS: system inflammatory reaction syndrome; MODS:multiple organ } \\
\text { dysfunction syndrome; HLH:hemophagocytic lymphohistiocytosis; CBP: continuous blood purification; ECMO: } \\
\text { extracorporeal membrane oxygenation; PIBO: post-infectious bronchiolitis obliterans. }\end{array}$} \\
\hline
\end{tabular}

\section{Stratified analysis according to timing of IVIg administration}

A total of 202 patients were stratified for analysis according to the timing of IVIg administration. No significant difference in demographic characteristics, clinical signs and symptoms, HAdVs type distribution, immunologic assays, and treatment before IVIg administration was observed between the two layers. Remarkably, among those laboratory findings on admission, lower hemoglobin, higher lactate dehydrogenase (LDH), and lower albumin levels were observed in later presenters. Furthermore, later presenters had higher fungal coinfection rate, more demands for mechanical ventilation, higher incidence of bronchiectasis, and longer fever duration in this study $(P<0.05)($ Table 1$)$. In particular, mortality was higher in later presenters $(6 / 74,8.11 \%)$ than that in early presenters $(4 / 128,3.31 \%)$, although the result was not statistically significant $(P=0.1756)$.

\section{Clinical responses to different dosages of IVIg administration in each layer}


With further analysis of each layer of patients, no statistically significant difference was observed in demographic characteristics, laboratory findings, pulmonary complications, and treatments before IVIg administration between the two groups according to different IVIg dosages (Table 2). When we analyzed primary end-points according to different dosages of IVIg administration, no statistically significant differences were found in the demand for advanced life support and outcomes for early presenters; therefore, it was considered as secondary end-points $(P>$ 0.05). For later presenters, results revealed shorter duration of fever and lower use of ECMO in high-dosage group than that in the low-dosage group $(P<0.05)$. However, among secondary end-points of later presenters, incidences of PIBO and bronchiectasis were not significantly different between the two groups $(P>0.05)$ (Table 3). 
Table 2

Clinical characteristics of children with severe adenovirus pneumonia before taking different dosage of IVIg in the study

\section{Variables}

Early presenters

\section{Demographic}

Age (months),

median(range)

Gender, male (\%)

Laboratory findings ${ }^{a}$

White cells $\left(\times 10^{\wedge} 9 / \mathrm{L}\right)$

(5-12),

median(range)

Neutrophil(× 10^9/L)

(5-12),

median(range)

Hemoglobin(g/L)

(105-

145),median(range)

Platelet $\left(\times 10^{\wedge} 12 / \mathrm{L}\right)$

(5-12),

median(range)

$\operatorname{HsCRP}(\mathrm{mg} / \mathrm{L})(<5)$

median(range)

LDH(U/L) (159-322)

median(range)

Albumin(g/L)(40-

50), median(range)

$\mathrm{PaO}_{2}(\mathrm{kPa})(\geq 10.9)$,

median (range)

$\mathrm{PaCO}_{2}(\mathrm{kPa})(4.66-$

5.99), median (range)

HAdV-3, No. (\%)

HAdV-7, No. (\%)

Co-infection ${ }^{b}$

Backteria, №. (\%)

Virus(except for

HAdVs), No. (\%)

Mycoplasmal

pneumonia, №. (\%)

$108.5(66-\quad 106(56-134) \quad 0.4341$

145)

259.5(70-

1180)
15.29(0.50- 20.16(0.46-

182.92)

631(210- 554.5(314-

3440)

33.40(21.22-

47.00)

4836)

$9.10(4.51-$

28.00)

$4.90(3.20-$

$6.74)$

8(11.11)

48(66.67)

47.40)

21.45)

$4.80(3.60-$

$6.70)$

$7(12.50)$
29(51.79)

20(27.78) 22(39.29)

10(13.89) 13(23.21)

20(27.78) $\quad$ 10(17.86)
High-dosage Low-dosage $P$

$(n=72) \quad(n=56)$

$12(3-96)$

41(56.94)

33(58.93)

0.8583

$34(72.34)$

15(55.56)

0.2018

\section{Later presenters}

High-dosage Low-dosage $P$

$(n=47) \quad(n=27)$

$\begin{array}{llllll}8.70(2.10- & 7.60(1.30- & 0.1497 & 6.20(1.50- & 7.60(1.60- & 0.5191 \\ 25.30) & 25.10) & & 30.60) & 21.60) & \end{array}$

$\begin{array}{llllll}4.96(0.651- & 4.005(0.48- & 0.1917 & 2.94(0.64- & 4.01(0.66- & 0.6136 \\ 21.84) & 19.21) & & 22.35) & 12.63) & \end{array}$

$227(81-1122) \quad 0.2666$

285(15-651)

$277(65-766)$

0.8913

0.7268

0.1971

$34.10(23.40-\quad 0.4493$

8.515(4.70- $\quad 0.0793$

0.4251

0.9999

0.1032

$33(70.21)$

$19.10(0.52-$

142.91)

953(260-

3767)

31.20(23.60-

41.10)

$8.30(4.10-$

20.07)

4.96(3.61-

7.54)

7(14.89)

$5(18.52)$

18(66.67)

$16.00(0.50-$

222.22)

668(272-

4570)

45.20)

9.365(3.30-

15.60)

4.85(3.61-

7.90)

0.4162

0.7705

0.8719

$30.25(20.20-$

0.3788

0.2633

0.6251

0.7485

0.1880

14(29.79)

9(33.33)

0.7977

0.2457

9(19.15)

8(29.63)

0.3911

0.2127

15(31.91)

7(25.93)

0.7921 


\begin{tabular}{|c|c|c|c|c|c|c|}
\hline \multirow[t]{2}{*}{ Variables } & \multicolumn{3}{|c|}{ Early presenters } & \multicolumn{3}{|c|}{ Later presenters } \\
\hline & $\begin{array}{l}\text { High-dosage } \\
(n=72)\end{array}$ & $\begin{array}{l}\text { Low-dosage } \\
(n=56)\end{array}$ & $\begin{array}{l}P \\
\text { value }\end{array}$ & $\begin{array}{l}\text { High-dosage } \\
(n=47)\end{array}$ & $\begin{array}{l}\text { Low-dosage } \\
(n=27)\end{array}$ & $\begin{array}{l}P \\
\text { value }\end{array}$ \\
\hline Fungi, No. (\%) & 6(8.33) & $7(12.50)$ & 0.5581 & $10(21.28)$ & $6(22.22)$ & 0.9999 \\
\hline \multicolumn{7}{|l|}{$\begin{array}{l}\text { Pulmonary } \\
\text { complications }^{b}\end{array}$} \\
\hline $\begin{array}{l}\text { Consolidation, No. } \\
(\%)\end{array}$ & $49(68.06)$ & $44(78.57)$ & 0.2317 & $38(80.85)$ & 19(70.37) & 0.3911 \\
\hline $\begin{array}{l}\text { Pleural effusion, No. } \\
(\%)\end{array}$ & 19(26.39) & $18(32.14)$ & 0.5566 & $16(34.04)$ & $9(33.33)$ & 0.9999 \\
\hline $\begin{array}{l}\text { Pneumothorax and } \\
\text { pneumomediastinum, } \\
\text { No. (\%) }\end{array}$ & $3(4.17)$ & $3(5.36)$ & 0.9999 & $3(6.38)$ & $4(14.81)$ & 0.2498 \\
\hline \multicolumn{7}{|l|}{$\begin{array}{l}\text { Treatment(before } \\
\text { IVlg) }\end{array}$} \\
\hline Antibotics, No. (\%) & $38(52.78)$ & $30(53.57)$ & 0.9999 & $30(63.83)$ & $17(62.96)$ & 0.9999 \\
\hline $\begin{array}{l}\text { Systemic } \\
\text { corticosteroid, No. (\%) }\end{array}$ & $48(66.67)$ & $30(53.57)$ & 0.1474 & $33(68.75)$ & $14(51.85)$ & 0.2135 \\
\hline \multicolumn{7}{|c|}{ Abbreviation:HsCRP: high-sensitivity C-reactive protein; LDH: lactate dehydrogenase; } \\
\hline \multicolumn{7}{|c|}{$\begin{array}{l}\text { aData extracted from the first test for } \\
\text { the childrens at admission; }\end{array}$} \\
\hline $\begin{array}{l}{ }^{b} \text { Data extracted from } t \\
\text { course of the patients. }\end{array}$ & whole & & & & & \\
\hline
\end{tabular}




\begin{tabular}{|c|c|c|c|c|c|c|}
\hline \multirow[t]{3}{*}{ Observation items } & \multicolumn{3}{|c|}{ Early presenters } & \multicolumn{2}{|c|}{ Later presenters } & \multirow{3}{*}{$\begin{array}{l}\mathrm{P} \\
\text { value } \\
\mathrm{P} \\
\text { value }\end{array}$} \\
\hline & $\begin{array}{l}\text { High- } \\
\text { dosage }\end{array}$ & $\begin{array}{l}\text { Low- } \\
\text { dosage }\end{array}$ & $\begin{array}{l}\mathrm{P} \\
\text { value }\end{array}$ & $\begin{array}{l}\text { High- } \\
\text { dosage }\end{array}$ & $\begin{array}{l}\text { Low- } \\
\text { dosage }\end{array}$ & \\
\hline & $(n=72)$ & $(n=56)$ & & $(n=47)$ & $(n=27)$ & \\
\hline \multicolumn{7}{|l|}{ Primary endpoints } \\
\hline Mechanical ventilation, No. (\%) & $17(23.61)$ & $9(16.07)$ & 0.3771 & 15(31.91) & $10(35.71)$ & 0.8026 \\
\hline CBP, No. (\%) & $3(4.17)$ & $2(3.57)$ & 0.9999 & $4(8.51)$ & $2(7.41)$ & 0.9999 \\
\hline ECMO, No. (\%) & $4(5.56)$ & $5(8.93)$ & 0.5032 & $1(2.13)$ & $5(18.52)$ & 0.0221 \\
\hline $\begin{array}{l}\text { Fever duration(days), } \\
\text { median(range) }\end{array}$ & $11(3-58)$ & $\begin{array}{l}11.5(6- \\
34)\end{array}$ & 0.1709 & $18(7-30)$ & $21(6-44)$ & 0.0293 \\
\hline $\begin{array}{l}\text { Length of stay(days), } \\
\text { median(range) }\end{array}$ & $13(4-46)$ & $13(7-70)$ & 0.8165 & $17(2-49)$ & $16(1-60)$ & 0.8055 \\
\hline Mortality, No. (\%) & $2(2.78)$ & $2(3.57)$ & 0.9999 & $2(4.26)$ & $4(14.81)$ & 0.1852 \\
\hline \multicolumn{7}{|l|}{ Secondary endpoints } \\
\hline PIBO, No. (\%) & 12(16.67) & $16(28.57)$ & 0.1326 & $11(23.40)$ & $7(25.93)$ & 0.9999 \\
\hline Bronchiectasis, No. (\%) & $1(1.39)$ & $1(1.79)$ & 0.9999 & $4(8.51)$ & $3(11.11)$ & 0.7008 \\
\hline
\end{tabular}

\section{Infusions and adverse events}

A total of 589 infusions were administered to 202 patients: 236 for the high-dosage group and 353 for the low-dosage group. Adverse events definitely, probably, or possibly related to the IVIg occurred during 39 of 589 infusions (6.62\%).

Adverse events complicating $\geq 1 \%$ of infusions were flushing $(12 / 589,2.04 \%)$, fever $(7 / 589,1.19 \%)$, and chills $(6 / 589$, $1.02 \%)$. No significant difference was observed in the incidence rate of adverse events between the two groups ( $P>$ 0.05) (Table 4). No serious adverse events were attributed to IVIg administration. 
Table 4

Adverse effects of children with severe adenovirus pneumonia in the study according to different dosage of IVIg

\begin{tabular}{|lllll|}
\hline Adverse effects & $\begin{array}{l}\text { Total } \\
(\mathbf{n}=\mathbf{5 8 9})\end{array}$ & $\begin{array}{l}\text { High-dosage } \\
(\mathbf{n}=\mathbf{2 3 6})\end{array}$ & $\begin{array}{l}\text { Low-dosage } \\
(\mathbf{n}=\mathbf{3 5 3})\end{array}$ & $\boldsymbol{P}$ value \\
\hline Flushing, No. (\%) & $12(2.04)$ & $8(3.36)$ & $4(1.13)$ & 0.0745 \\
\hline Headache, No. (\%) & $2(0.34)$ & $0(0.00)$ & $2(0.57)$ & 0.5189 \\
\hline Fever, No. (\%) & $7(1.19)$ & $3(1.27)$ & $4(1.13)$ & 0.9999 \\
\hline Chills, No. (\%) & $6(1.02)$ & $2(0.85)$ & $4(1.13)$ & 0.9999 \\
\hline Nausea and Vomiting, No. (\%) & $5(0.85)$ & $2(0.85)$ & $3(0.85)$ & 0.9999 \\
\hline Hypotension, No. (\%) & $1(0.17)$ & $1(0.54)$ & $0(0.00)$ & 0.4007 \\
\hline Nephrotoxicity, No. (\%) & $0(0.00)$ & $0(0.00)$ & $0(0.00)$ & 1.0000 \\
\hline Myalgias, No. (\%) & $1(0.17)$ & $1(0.42)$ & $0(0.00)$ & 1.0000 \\
\hline Anaphylactic shock, No. (\%) & $0(0.00)$ & $0(0.00)$ & $0(0.00)$ & 1.0000 \\
\hline Thromboembolic events, No. (\%) & $0(0.00)$ & $0(0.00)$ & $0(0.00)$ & 1.0000 \\
\hline Hemolysis, No. (\%) & $0(0.00)$ & $0(0.00)$ & $0(0.00)$ & 1.0000 \\
\hline Fatigue and Lethargy, No. (\%) & $5(0.85)$ & $2(0.85)$ & $3(0.85)$ & 0.9999 \\
\hline Total & $39(6.62)$ & $19(8.05)$ & $20(5.67)$ & 0.3103 \\
\hline
\end{tabular}

\section{Discussion}

Adenovirus pneumonia is a pulmonary infectious disease commonly occurring in children[4,23]. Most patients have mild disease and recover from adenovirus pneumonia, but severe adenovirus pneumonia can result in considerable morbidity and mortality in immunocompetent pediatric patients[3]. HAdV-7 is known to be associated with serious pneumonia in children and is more prone to be followed by the PIBO development [24]. However, the standard therapy for the management of severe adenovirus pneumonia is not yet established to date, and efficacy data evaluating possible treatment options were not systematically studied in non-immunocompromised pediatric patients. In this retrospective study of 202 patients treated with intravenous immunoglobulin (IVIg) for severe adenovirus pneumonia at our hospital, we preliminarily assessed the potential efficacy and safety of the treatment in pediatric patients.

IVlg was used for non-immunocompromised children with severe adenovirus pneumonia when they combined serious complications[23]. The principal components of IVIg are natural antibodies (NAbs) of the IgG isotype. NAbs can enhance the recruitment of virus into lymphoid organs where they are present to $T$ and $B$ cells, thereby eliciting an active immune response[25]. The protective effect of IVIg against infections has been attributed to the ability of specific antibodies to neutralize pathogens[26]. Indeed, research has found that commercial IVIg preparations possess anti-adenovirus activity in vitro, suggesting a potential role for adjuvant IVlg in adenovirus infectious diseases[14]. In this retrospective study, IVIg was administered as supplementary therapy to suppress viral replication through neutralization by viral antibodies. The stratification analysis by illness duration according to IVIg administration showed that later presenters were more associated with lower hemoglobin and albumin levels and higher LDH levels, and more importantly, fungal coinfections were more prevalent in these patients. These results 
indicate that later presenters have been more complicated and serious. Based on the analysis results, early administration of IVIg was important to decrease the use of mechanical ventilation and shorten the fever duration. For the long-term outcome, early treatment was also crucial to lowering the incidence rate of bronchiectasis. In particular, early presenters had lower mortality than later presenters had, although the difference was not statistically significant. Therefore, the study confirmed the importance of early IVIg administration in non-immunocompromised children with severe adenovirus pneumonia.

The following step toward the therapeutic utilization of IVIg for pediatric severe adenovirus pneumonia would be the fine-tuning the dose regimen in a clinical study. In this study, patients were categorized into two groups based on their IVIg treatment regimens during each hospitalization: high-dosage group underwent IVIg treatment with $1 \mathrm{~g} / \mathrm{kg}$ for 2 days, whereas low-dosage group were treated with $0.4-0.5 \mathrm{~g} / \mathrm{kg}$ for 3-5 days. At high doses, IVIg inhibits the function of different arms of the immune system, including inhibition of the maturation and function of dendritic cells, attenuation of T-cell proliferation, and production of proinflammatory cytokines[26]. A previous study demonstrated that a high-dose regimen might not be appropriate for the treatment of infectious diseases. However, recent research has found that activation of the immune system and generation of numerous chemokines and cytokines could play a major role in the pathogenesis of adenovirus tissue damage[27]. In our study, no significant differences were observed between low- and high-dose groups in clinical end-points of early presenters. Nevertheless, for later presenters, the high-dosage group can reduce the fever duration and ECMO usage, although different dosages of IVIg administration had little impact on the prognosis of later presenters. In other words, later presenters with severe adenovirus pneumonia could benefit from a high-dose of regimen IVIg during hospitalization.

The intravenous immunoglobulin level in this study was well tolerated, and no serious adverse events were found to be attributed to IVIg. Currently, most viewpoints are that majority of adverse effects are associated with high IVIg dosage[16]. Moreover, the incidence was $6.62 \%$ during infusion with IVIg in this study. Furthermore, the IVIg dosage was not related with the incidence of adverse events in the two study groups. Thus, different dosages to guarantee the efficacy of therapy and minimize adverse effects were observed in our study.

Limitations of our study were mainly due to its retrospective file review design, with all associated disadvantages of this study type (e.g., missing information, poor documentation, etc.). However, this study is the first to explore the timing and dosage of IVIg administration in non-immunocompromised pediatric patients with severe adenovirus pneumonia and to evaluate the correlation between different dosages and the incidence of adverse events.

\section{Conclusions}

IVIg is an adjunctive therapy for the treatment of severe adenovirus pneumonia in children. Our data affirm that early IVIg administration for severe adenovirus pneumonia in non-immunocompromised patients could be a treatment strategy worth considering. For later presenters, a high dosage of IVIg is effective in reducing the ECMO use and fever duration, which is probably beneficial in these patients.

\section{Abbreviations}

IVIg: intravenous immunoglobulin; HAdVs:human adenoviruses; RTIs:respiratory tract infections; PIBO:post-infectious bronchiolitis obliterans; CDV:cidofovir; PCR:polymerase chain reaction; PICU:pediatric intensive care unit; CBP:continuous blood purification; ECMO:extracorporeal membrane oxygenation; LOS:length of stay; ARDS:Acute respiratory distress syndrome; MODS:multiple organ dysfunction syndrome; HLH:hemophagocytic lymphohistiocytosis; Nabs:natural antibodies

Page 13/18 


\section{Declarations}

\section{Availability of data and materials}

The dataset generated for this study will be made available by the corresponding author on request in an anonymized version to guarantee low risk of patient identification.

\section{Ethics approval and consent to participate}

The study was approved by Ethics Committee of Guangzhou Women and Children's Medical Center of Guangzhou Medical University. The study does not include patients' private information, has no effects on the routine diagnosis and treatment of patients, patients do not participate in the study for additional tests or examinations.

\section{Consent for publication}

The written informed consent to publish for all the subjects in this study was provided by their parents, guardians, or next of kin.

\section{Competing interests}

The authors declare no conflicts of interests relevant to this article.

\section{Funding}

The study was approved by Guangzhou Municipal Science and Technology Bureau, China [Nos. 202002030061].

\section{Authors' contributions}

HF and CC analyzed the data and wrote the manuscript. XX and SZ collected the data. GY and WJ followed up the prognosis. GL performed the study design and critical revision. All authors report no actual or potential conflicts of interests.

\section{Acknowledgments}

We are very appreciative to the children and their families.

\section{References}

1. Lynch JP 3rd, Kajon AE. Adenovirus. Epidemiology, Global Spread of Novel Serotypes, and Advances in Treatment and Prevention. Seminars in respiratory critical care medicine. 2016;37(4):586-602. https://doi.org/10.1055/s-0036-1584923.

2. Lee J, Choi EH, Lee HJ. Comprehensive serotyping and epidemiology of human adenovirus isolated from the respiratory tract of Korean children over 17 consecutive years (1991-2007). J Med Virol. 2010;82(4):624-31. https://doi.org/10.1002/jmv.21701. 
3. Wu P-Q, Zeng S-Q, Yin G-Q, Huang J-J, Xie Z-W, Lu G, et al. Clinical manifestations and risk factors of adenovirus respiratory infection in hospitalized children in Guangzhou, China during the 2011-2014 period. Medicine. 2020;99(4):e18584. https://doi.org/10.1097/md.0000000000018584.

4. Shen C-F, Wang S-M, Ho T-S, Liu C-C. Clinical features of community acquired adenovirus pneumonia during the 2011 community outbreak in Southern Taiwan: role of host immune response. BMC Infect Dis. 2017; 17(1). https://doi.org/10.1186/s12879-017-2272-5.

5. $26: 41-8$

https://doi.org/10.1016/j.prrv.2017.06.013

Tramper-Stranders GA. Childhood community-acquired pneumonia: A review of etiology- and antimicrobial treatment studies. Paediatr Respir Rev. 2018; 26:41 - 8. https://doi.org/10.1016/j.prrv.2017.06.013.

6. Murtagh P, Giubergia V, Viale D, Bauer G, Pena HG. Lower respiratory infections by adenovirus in children. Clinical features and risk factors for bronchiolitis obliterans and mortality. Pediatric pulmonology. 2009;44(5):450-6. https://doi.org/10.1002/ppul.20984.

7. Leung AKC, Wong AHC, Hon KL. Community-Acquired Pneumonia in Children. Recent patents on inflammation \& allergy drug discovery. 2018; 12(2):136-44. https://doi.org/10.2174/1872213X12666180621163821.

8. Naesens L, Lenaerts L, Andrei G, Snoeck R, Van Beers D, Holy A, et al. Antiadenovirus activities of several classes of nucleoside and nucleotide analogues. Antimicrob Agents Chemother. 2005;49(3):1010-6. https://doi.org/10.1128/AAC.49.3.1010-1016.2005.

9. Ganapathi L, Arnold A, Jones S, Patterson A, Graham D, Harper M, et al. Use of cidofovir in pediatric patients with adenovirus infection. F1000Research. 2016;5:758. https://doi.org/10.12688/f1000research.8374.2.

10. Prina E, Ceccato A, Torres A. New aspects in the management of pneumonia. Critical care. 2016;20(1):267. https://doi.org/10.1186/s13054-016-1442-y.

11. Serrano RM, Darragh RK, Parent JJ. Successful treatment of disseminated adenovirus infection with cidofovir and intravenous immunoglobulin in an infant following heart transplant. Cardiol Young. 2018;28(6):888-9. https://doi.org/10.1017/S1047951118000379.

12. I.V. immunoglobulin therapy for infectious diseases. Drug Ther Bull. 2010;48(5):57-60. https://doi.org/10.1136/dtb.2009.07.0032.

13. Crowley JC, Gropper MA. IV Immunoglobulin: A Useful Tool for the Severe Pneumonia Toolbox? Critical care medicine. 2016;44(1):250-1. https://doi.org/10.1097/CCM.0000000000001334.

14. Krause I, Wu R, Sherer Y, Patanik M, Peter JB, Shoenfeld Y. In vitro antiviral and antibacterial activity of commercial intravenous immunoglobulin preparations-a potential role for adjuvant intravenous immunoglobulin therapy in infectious diseases. Transfusion medicine. 2002;12(2):133-9. https://doi.org/10.1046/j.1365-3148.2002.00360.x.

15. Li MY, Kelly J, Subhi R, Were W, Duke T. Global use of the WHO pocket book of hospital care for children. Paediatrics international child health. 2013;33(1):4-17. https://doi.org/10.1179/2046905512Y.0000000017.

16. Guo Y, Tian X, Wang X, Xiao Z. Adverse Effects of Immunoglobulin Therapy. Frontiers in immunology. 2018;9:1299. https://doi.org/10.3389/fimmu.2018.01299.

17. Elajez R, Ezzeldin A, Gaber H. Safety evaluation of intravenous immunoglobulin in pediatric patients: a retrospective, 1-year observational study. Ther Adv Drug Saf. 2019;10:2042098619876736. https://doi.org/10.1177/2042098619876736.

18. Orbach H, Katz U, Sherer Y, Shoenfeld Y. Intravenous immunoglobulin: adverse effects and safe administration. Clin Rev Allergy Immunol. 2005;29(3):173-84. https://doi.org/10.1385/CRIAl:29:3:173. 
19. Pediatric Acute Lung Injury Consensus Conference G. Pediatric acute respiratory distress syndrome: consensus recommendations from the Pediatric Acute Lung Injury Consensus Conference. Pediatr Crit Care Med. 2015; 16(5):428 - 39. https://doi.org/10.1097/PCC.0000000000000350.

20. Weiss SL, Peters MJ, Alhazzani W, Agus MSD, Flori HR, Inwald DP, et al. Surviving Sepsis Campaign International Guidelines for the Management of Septic Shock and Sepsis-Associated Organ Dysfunction in Children. Pediatr Crit Care Med. 2020;21(2):e52-106. https://doi.org/10.1097/PCC.0000000000002198.

21. Leteurtre S, Martinot A, Duhamel A, Proulx F, Grandbastien B, Cotting J, et al. Validation of the paediatric logistic organ dysfunction (PELOD) score: prospective, observational, multicentre study. Lancet. 2003;362(9379):192-7. https://doi.org/10.1016/S0140-6736(03)13908-6.

22. Jordan MB, Allen CE, Greenberg J, Henry M, Hermiston ML, Kumar A, et al. Challenges in the diagnosis of hemophagocytic lymphohistiocytosis: Recommendations from the North American Consortium for Histiocytosis (NACHO). Pediatr Blood Cancer. 2019;66(11):e27929. https://doi.org/10.1002/pbc.27929.

23. Foong Ng K, Kee Tan K, Hong Ng B, Nair P, Ying Gan W. Epidemiology of adenovirus respiratory infections among hospitalized children in Seremban. Malaysia Trans R Soc Trop Med Hyg. 2015;109(7):433-9. https://doi.org/10.1093/trstmh/trv042.

24. Castro-Rodriguez JA, Daszenies C, Garcia M, Meyer R, Gonzales R. Adenovirus pneumonia in infants and factors for developing bronchiolitis obliterans: a 5-year follow-up. Pediatric pulmonology. 2006;41(10):947-53. https://doi.org/10.1002/ppul.20472.

25. Ochsenbein AF, Fehr T, Lutz C, Suter M, Brombacher F, Hengartner $H$, et al. Control of early viral and bacterial distribution and disease by natural antibodies. Science. 1999;286(5447):2156-9. https://doi.org/10.1126/science.286.5447.2156.

26. Bayry J, Lacroix-Desmazes S, Kazatchkine MD, Kaveri SV. Intravenous immunoglobulin for infectious diseases: back to the pre-antibiotic and passive prophylaxis era? Trends Pharmacol Sci. 2004;25(6):306-10. https://doi.org/10.1016/j.tips.2004.04.002.

27. Chen RF, Lee CY. Adenoviruses types, cell receptors and local innate cytokines in adenovirus infection. Int Rev Immunol. 2014;33(1):45-53. https://doi.org/10.3109/08830185.2013.823420.

\section{Figures}




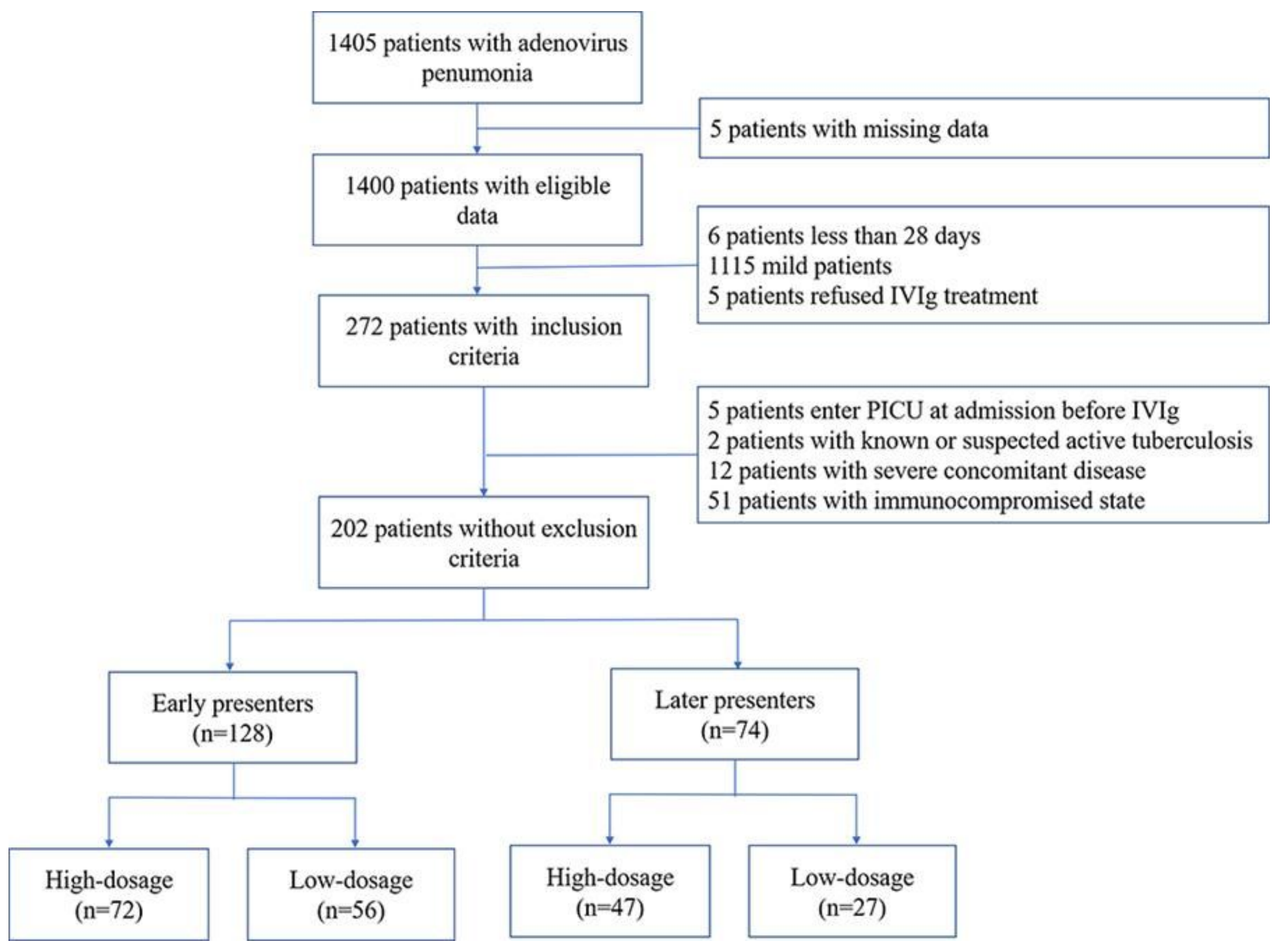

Figure 1

Study flowchart 

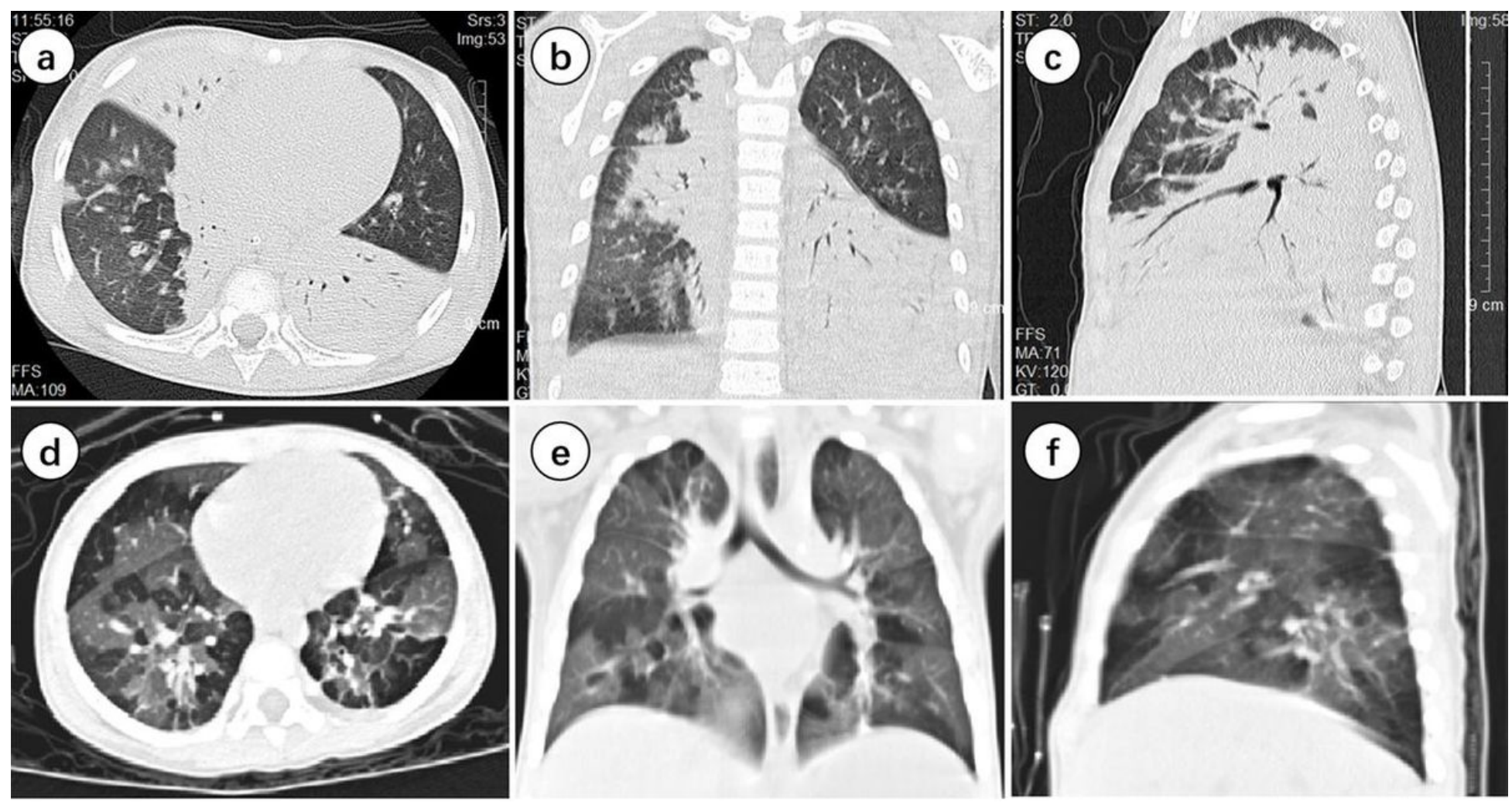

Figure 2

Imaging features of severe adenovirus pneumonia. High-resolution CT scan of the chest revealing diffuse infiltration and consolidation areas in the right upper and left lower lobes of a 4-year-old girl(a, b, c). High-resolution CT scan of the chest revealing mosaic sign in lung fields in a 1-year-old boy(d, e, f) .

\section{Supplementary Files}

This is a list of supplementary files associated with this preprint. Click to download.

- supplementarydata.xIsx 